\title{
Clinical Empathy Training: An Explorative Qualitative Study in Search of the Barriers and Facilitators of Implementation
}

Luca Claudia Barak ( l.c.barak@olvg.nl )

OLVG Hospital

Giliam Kuijpers

Social enterprize Medgezel

Lotte Hoeijmakers

OLVG Hospital

\section{Fedde Scheele}

Vrije Universiteit Amsterdam

Research Article

Keywords:

Posted Date: March 7th, 2022

DOI: https://doi.org/10.21203/rs.3.rs-1357849/v1

License: (1) This work is licensed under a Creative Commons Attribution 4.0 International License. Read Full License 


\section{Abstract}

Background: Amid concerns about the decline of empathy during clinical training of medical clerks, evidence that empathy improves patient outcomes suggests some potential for teaching empathy in ways that will affect the knowledge, attitude and behaviour of medical clerks. This potential alone cannot, however, guarantee the success of educational innovations to introduce empathy to the medical curriculum. This research aims to investigate an implementation failure of a specific clinical initiative to enhance the empathy skills of clerks, namely the training of clerks to act as 'MedGezels' or 'medical coaches'.

Method: We conducted an explorative qualitative study on the basis of interview data collected and analyzed in a manner inspired by grounded theory and viewed through the lens of the readiness for change theory. We conducted semi-structured interviews with the most important stakeholders in the particular case study. Thematic analysis was done based on open and axial coding using ATLAS.ti 9, a qualitative data analyzing software program, which facilitated the emergence of common themes of interest for the study.

Results: A total of 13 relevant stakeholders participated as interviewees in our study. The data was collected over a period of 3 months, from April to June 2021. Our analysis generated 6 main themes which can provide insights into why the implementation of the MedGezel educational innovation failed so far. The themes that emerged were: the case for change: why change?; practical necessity; leadership; management and resources; staff culture and the alignment with the corporate strategy.

Discussion: The implementation failure is partially explainable as resulting from the personal attitudes and choices of participants who struggled to reconcile a vision that they liked but whose side effects they feared. While participants mentioned management and leadership issues several times, the importance of these was downplayed given that such organizational issues could be easily resolved in practice. What was more important and fatal to the initiative was its lack of alignment with staff culture, despite its alignment with corporate strategy.

Conclusion: This investigation into the reasons behind the implementation failure of the MedGezel program produced 6 explanatory themes of which staff culture was most convincing.

\section{Background}

There are concerns about the decline of empathy during clinical training of medical clerks (1). This research focusses on the readiness for change of relevant stakeholders around a practical initiative to enhance especially empathy skills of physicians.

The time and energy spent on teaching humanism in care, characterized by interest in and respect for the patient's concerns and values regarding psychological, social and spiritual domains, has to be balanced 
with the need of medical expertise to deal with the complexity of large interprofessional teams and finding ways to cope with issues of high workload and serious time pressure in health systems (2).

There is evidence that empathy, a hallmark of humanistic care, improves patient outcomes $(1,3,4)$, and that the teaching of empathy has an effect on the knowledge, attitude and behaviour of medical clerks (5). As we make more attempts to implement empathy in the formal undergraduate curriculum, it is important to understand the actual clinical practice as well. The medical world is characterized by a powerful and intensive socialization process that has sometimes been referred to as the "hidden curriculum" (2). This reveals itself in informal forms of control over group functioning, where unwritten rules structure inter-personal manners and relations of authority (6). This hidden curriculum is highly influential, and may easily overrule knowledge gained and attitudes achieved in previous course-work, and furthermore shape future attitudes and behaviours of a clerk thereafter (7). It is through this hidden curriculum that a specific professional culture is transferred and maintained, and because it is shown in so many different times and places it is possible to speak of a common professional identity, or the medical "habitus" $(6,8)$. In the practical initiative under study in this manuscript, attention for empathic health care may ask for a more prominent position of empathy within the medical habitus.

Branch et al. provided practical tips on how to establish a climate of humanism by: recognizing and using learning opportunities; using role modelling; making sure that the learners are actively engaged; being practical and relevant; and using multiple, up-to-date strategies at the same time. From the perspective of change management, this approach of Branch et al. is attractive, since no structural change in the clinical workflow is demanded and the alignment is relatively good (9). In our opinion, optimally engaging clerks as part of the health care team (10) by making them stewards of humanistic care, is a sensible further step to take. They need to become practicing, engaged and responsible team members (10), they need to be reflective of their actions and on the humanistic care climate that they are part of (11), and finally they need to rehearse their roles (5). Based on these principles, we decided to train clerks as medical coaches and to prepare them for a responsible role as steward of humanistic care. Our focus for the learning effect is on empathy and person-centered care (see Box 1). For the pilot a coordinator was assigned to assist in explanation and planning at the outpatient clinics.

\section{Box 1. MedGezel}

In the last few years, we have created and tested a prototype of an educational intervention to teach medical clerks empathic behaviours by acting as a medical coach (a MedGezel), guiding a patient in obtaining a patient-centered doctor's consultation. More than 100 medical students selected from all the medical faculties in the Netherlands, were voluntarily trained as medical coaches and guided approximately 100 patients during their care trajectory in several Dutch hospitals. Special emphasis was on compassionate interactions with the patients by deep listening and personal attention to the lives of the patients.

A coach empowers the intrinsic ability of people to take charge of their wishes, needs and challenges (36) on the understanding that people reach their own answers to their personal challenges. 
To become a coach for others you have to first look at your own beliefs, wishes and challenges (37). As such, our medical coach training consists of two crucial components: getting to know yourself and getting to know the patient.

After the medical coach training, the MedGezel prototype of empowering patients consists of three stages: 1: before the consultation with the doctor, the medical students connect with the patients to discuss their personal wishes, needs and challenges. The medical students prepare the patients to the consultation by rehearsing the most important questions. 2: During the consultation with the medical specialist, the medical students act as a patient advocate and buddy of the patient. 3: After the consultation the medical student repeat the most prominent features of the dialogue with the medical specialist and the students make sure that everything is well understood by the patients. They also make sure that the feelings and concerns of the patient are met with dignity and respect.

As collateral gains of deploying a MedGezel, patients may feel better heard and better prepared for their appointments, which gives physicians more opportunities for patient-centered care.

At the start of the pilot, we were met with enthusiasm from multiple academic and affiliating training hospitals, a national health innovation prize was awarded (2020), and national media reported positively about the project. However, when the time came to make concrete agreements with hospital departments on how to diffuse of this innovation, we hit "a wall of goodwill". There is much goodwill in relation to the vision and the idea of training the medical clerks to become medical coaches. Furthermore, this practical innovation of the medical curriculum matches official policies concerning patient participation. Nevertheless, this "wall" that we encountered was characterized by hesitancy to actually move forward with the changes it proposed. Our hypotheses were that despite their enthusiasm and goodwill, stakeholders, the organization and/or the medical culture were not ready for this kind of change.

It is important in this current period of transition in health care (12) as well as in the training pedagogy that tries to prepare for it (13) to understand problems that arise when implementing challenging educational innovations in the medical curriculum. This is especially the case when these innovations do not entirely align with current practice (9) and even ask for a change in the medical habitus (8). As such, it is necessary to know why change programs get implemented or not. According to the theory of readiness for organizational change, an innovation needs to satisfy a specific need and add value to the context, by which it needs to be adopted and routinized into standard practice (14). To create readiness, a sense of urgency needs to be established, team members need to be empowered, and an appealing vision must be offered along with confidence that it can be realized. The implementation progress is accelerated in the presence of good leaders who are fully equipped with toolboxes of change management principles. Change is seen as a social process that requires creativity, a sense of ownership and support of multiple layers of organization (15). The concept of readiness for change may assist us to find out where the "wall of goodwill" in our case consists of and find out what its most important sources are. Medical education literature dealing with innovation failure in practice is scarce (16) and contributing to the literature of what can be learned from implementation successes and failures may add to our understanding of 
change management principles in the context of clinical learning processes. Learning from mistakes is a powerful instrument for advancement (17).

Our research question is: Which clarifications result from a readiness for change perspective on an implementation failure regarding practical empathy education for clerks in patient care. Better understanding of this "wall of goodwill" towards curriculum changes for empathy is especially important, because of the ongoing transition in healthcare towards patient-centered care.

\section{Methods}

\section{Setting}

This study focused on gaining knowledge of the factors that influence the "wall of goodwill" for the implementation of an empathy educational innovation. We conducted the study in the Netherlands, where the pilot of MedGezel took place in training hospitals.

\section{Study design}

Because this is an under-researched domain, we conducted an explorative qualitative interview study inspired by grounded theory and using the lens of the readiness for change theory. Grounded theory, first developed by Glaser and Strauss, involves an inductive but systematic approach to generate a conceptual framework or theory that is grounded in actively constructed data, to grow beyond static analysis to multiple layers of meaning $(18,19)$. Rich data has been collected in semi-structured interviews conducted with the most important stakeholders.

\section{Semi-structured interviews}

A semi-structured approach was chosen because it allows for more detailed responses and additional questions. It invites the interviewee to talk more about their own attitudes, beliefs, behaviors or experiences (18). We developed a tentative structure for a semi-structured interview based on the knowledge gained from a literature search, consistent with Glaser's approach of grounded theory (20). This generated the following topics for discussion: the implementation of patient-centered care, empathy in the consultation room and ideas concerning the implementation of the medical coach model. These topics were discussed from the perspective of the elements of change derived from the STORC questionnaire. The elements used in this questionnaire are: Pressure to change; Appropriateness; Necessity to change; management support an leadership; staff culture; the formal leader; involvement; Project resources; Clarity of mission and goals; and the implementation plan (21).

\section{Participants and sampling procedure}

To gain rich data and to widen the scope of this study, the perspective of different kinds of participants was sought, namely: both physicians who participated in the MedGezel pilot but also physicians who did 
not participate; educators and physicians who are responsible for education in their respective departments; managers involved in the educational infrastructure for medical clerks in clinical practice and marketers; and lastly medical clerks in their rotation phase of their training. Recruitment of participants continued until theoretical data saturation was reached.

\section{Data collection}

All semi-structured interviews were conducted by the main researcher (LB) online via ZOOM or in private offices between April 2021 and June 2021. Selected potential participants were contacted to explain the nature of the research, the aim of the study and the value of participation. An appointment was made and a letter for consent was asked to be signed before the interview. The interviews were audio-recorded, transcribed verbatim and reviewed for accuracy by the main researcher (LB). The audiotapes and transcriptions were anonymized for everyone except for the researcher and stored in a specifically designed safe environment for research data.

\section{Data analysis}

Thematical analysis was done based on open and axial coding using ATLAS.ti 9, a qualitative data analyzing software program. Repeated interim analysis (deductive) was used, which facilitated exploration of newly emerging issues in subsequent interviews (inductive), in an alternating inductive and deductive manner.

During the first phase of open coding, parts of the transcripts were coded with a label that summarized their contents. In a process of continuous comparison and interpretation of these labels, categories were then generated. This axial coding reduced the number of labels by clustering closely related labels into more comprehensive categories. During the last phase, based on the detected categories and informed by organizational readiness for change theory, themes emerged during discussions within the author team. During this alternatively inductive (collecting interview data) and deductive process (categorizing, themes detection) the level of abstraction increased and apparent relations between categories and themes became visible. After the first coding and categorization rounds, a second researcher was brought into the process and possible differences in the coding and categorization process were addressed until major consensus was reached. Being informed by the organizational readiness for change theory helped make the emerging themes more generalizable and increased confidence in the validity (18).

\section{Reflexivity}

Choices about identifying and selecting more complex categories and themes based on the grounded theory approach was done by: the main researcher, BSc and PhD-candidate Luca Barak; the second researcher, who helped in coding and categorizing, physician and manager of education, Lotte Hoeijmakers; co-investigator, physician, curriculum designer and trainer of medical coaches Giliam Kuijpers and Fedde Scheele, a physician and an expert in change theory. 


\section{Results}

A total of 23 relevant stakeholders were invited to participate in our study, of which 13 agreed. Of the included participants, 6 were specialists, 2 were clerks, 3 were educators and 2 were involved in quality departments. Of the specialists 4 had a double function, either with the quality department or with the undergraduate education. All of the participants held positions in one of four training hospitals, the OLVG hospital, Spaarne Gasthuis, the VUmc or the AMC. All interviews were conducted in Dutch, the language in which all the participants were highly proficient. The interviews lasted between 30 and 90 minutes each. The data was collected in a period of 3 months, from April to June 2021. After conducting 13 interviews, data saturation was reached, so there was no need to invite new participants.

Our analyses resulted in six main themes found within the concept of readiness for change which can provide insights into why the implementation of the MedGezel educational innovation failed. The themes that emerged were: the case for change: why change?; practical necessity; leadership; management and resources; staff culture and the alignment with the corporate strategy.

\section{The case for change: why change?}

The case for change in the form of MedGezel revolved around the need for a structured way of bringing more empathy and a better perspective on the patient journey to the training of future doctors. During our interviews we tried to discover participant perceptions regarding the MedGezel proposition. Our results indicated that most of the participants perceived the proposition of a change towards a new empathic program as being a necessary innovation. They stated that the innovation brings several wins, such as the clerk learning to see the patients' viewpoint, the patients feeling more seen and heard, and the specialist knowing what matters the most for the patient, as evidence in the following quotes:

"We saw the needs on both sides; with the patients, but also that it's good in the training for clerks, because we also know that there are clerks who find it quite difficult to think from the patient's point of view."

"I strongly believe that it has added value for the patients, because there is someone sitting next to them who also has a bit of medical knowledge and who prepares the consultation with the patient."

"It is always nice for the specialist if there is a clear question, because otherwise we will have to fill it in, and that might not always be correct."

The first impression, therefore, was that the proposed innovation elicited general goodwill, although some participants expressed second thoughts. A minority of the participants argued against the necessity of change towards more empathy in the medical education. These participants indicated that other ongoing similar initiatives to make doctors more patient oriented made the innovation unnecessary: "We are already listening very well and making decisions together, so why should we still use a MedGezel or why should we do a training "deciding together"?" 
Another participant said that sometimes empathy is not the main goal of the doctor's consultation. This is especially the case with consultations with new patients where the main goal is to find out as much as possible about the medical problems and at those moments little time is available for an empathic conversation. "At some point you have a half hour for taking your medical history, doing your physical examination and that's it. Empathy is not the purpose of that consultation."

Some participants argued that the decline of empathy is inevitable and was something that had to be accepted. They said that this might be due to the more difficult medical tasks a clerk or a freshly graduated doctor has to perform. Also, getting used to the difficult situations, such as seeing a lot of patients over time with the same terrible conditions, might make the experience less special and decrease the empathy of a doctor. One physician made the comparison to newly licensed drivers: "You forget that a patient is vulnerable. When you are a young doctor, you're much more concerned with switching, coupling in first gear, second gear, indicating directions. That is very different from surveying a traffic situation, whilst that might be the most important for a patient."

An educator said that strong competition within educational departments creates a fear of losing hardfought time on the program whenever an innovation is introduced. Therefore, they might not regard empathy education as a priority. "That is the thing with patient perspective educational programs, they meander against the borders of medical ethics, medical psychology and also the educational curriculum of professional conduct. They all have to fight with every curriculum innovation to defend their hours in the curriculum training."

One of the specialists questioned if empathy is even teachable, because feeling empathic is not the same as showing empathy. "You can't teach empathy, it's in you. This means that some people are more and others less suitable for the profession."

To summarize, among the participants, most had a positive attitude towards this innovation in the form of MedGezel. However, many conditions were created to question the necessity of the innovation. Moreover, some extenuated the fact that the innovation had not been implemented yet.

\section{Practical necessity}

Most of the participants recognized the need for a medical coach to guide patients. They explained that the medical aspects around a patient can sometimes clouds a doctor's view on the patient's personal life:

The focus of the medical curriculum is directed on the medical aspect around a patient and the doctors view on a patient, it is less about the experiences of a patient and the world they live in.

"It is good for the clerk to see what happens when a patient walks out of the consultation room. What did he remember? What goes on in the patient? What kind of question will he have? We do not see that perspective."

Most participants thought that for clerks to learn the patient's perspective from the beginning of their training might bring advantages, not only for their education but also for their career as a future doctor. 
One of the clerks mentioned: "I will keep using some of the striking questions I learned, like "What entails a good day and a bad one?' and 'What do you prefer to do in your normal day life?'."

Furthermore, some of the participants mentioned that with a MedGezel patients would acquire a sparring partner with whom they could reflect their feelings and concerns: "Some of the patients are very assertive and had their feet wetted before, others do need a sparring partner and are now able to reflect their feelings with a clerk, who will learn from this as well."

Though the importance of a living coach who can interrupt and coach the patient, was mentioned by many participants, some questioned the necessity of the innovation regarding patient participation. In the educational department one of the participants said that there are already multiple educational programs that are more patient oriented: "There are already more educational components like "the clerk follows a patient journey"."

In their view, there were already digital questionnaires available through which more patient participation could be achieved in the consultation room: "At the moment, there is a new feature in EPIC (electronic patient file) that allows patients to send the doctor questions in advance. With this feature the patients are forced to think more about their questions ahead."

In balancing the pro's and con's, some argued that the timeinvestment of the MedGezel innovation would be quite high, which raised the question of what added value it would bring: "You have to be very critical, how much work does it cost us and what does it get us?"

One educator expressed worries that the clerkship itself might be threatened by adding yet another activity, given that a lot of time is already spent by clerks on extracurricular activities. Moreover, some expressed concerns that some clerks might feel disadvantaged for missing out on more technical teaching moments while working as a MedGezel: "The question remains: Will they miss something what the other clerks do get? They spend a lot of time on it, it was quite intensive."

Altogether, suggestions were made that while the MedGezel innovation could expand the deeper understandings of person behind the patient, it could also be relatively quite time-consuming, considering other priorities in the medical curriculum.

\section{Leadership}

Leaders are often needed to carry an innovation forward, and some participants said that such leaders would truly have to believe in the innovation in order to get others to join them: "In one of my projects the programdirectors were involved, both people with a great heart for education, who have a vision on education and if they are convinced that it makes sense, they will stand up for it."

Also, one of the specialists with a quality assignment argued that sometimes it helped if the leader was someone who was respected and had built credibility in past projects: "In the past we worked well 
together, that is important, you know each other so you will not start from zero. You will think: Ok, fine, trustworthy."

Most participants saw strong leadership as a possible facilitator to bring an innovation to the next level: "What works is, I call it a trinity: a clinical champion, a head of department and a kind of change agent, which could be a very enthusiastic senior nurse, who stands on a soapbox announcing new ideas."

\section{Management and resources}

Some departments encountered diminutive difficulties with the implementation of MedGezel. Those departments agreed that the added value was more important and were willing to face the obstacles in a way of making this implementation work: "At our department the question 'how do we solve this?' was addressed by starting with only a few clerks to understand the possible technical difficulties, having two highly involved staff members and a strong feeling of importance for this kind of empathic innovations."

Though for some departments the implementation encountered minor difficulties, most concerns mentioned by the other participants had to do with difficulties in the scheduling of patients and clerks:

"To fit in a MedGezel, a new schedule needs to be made, and who is going to see the patients as clerk when the clerk is working as a MedGezel at that point."

"Who is going to select the patients? And who is going to call these patients?"

A expert from the quality department stated that a clear format could overcome these mentioned difficulties: "The schedule-challenges could be solved with a format for the selection of the right patients and a new scheduling system within EPIC." Also, this expert sugested starting a project group with people who work as outclinic assistants or secretairies in order to really understand what happens on the workfloor: "It is very important to have a projectgroup with people who really understand the workproces in the clinic."

Another concern was raised about financial contributions for the training of the medical coaches: "Where will the money come from? Because somebody has to invest, the hospital or the department?"

Even though the importance of empathy was clear to the majority of the participants, nearly all participants observed an organizational challenge in implementing the innovation: "With how the clerkship is organized now, we do not encounter a "not wanting", but rather a organizational implementation-challenge."

\section{Staff culture}

During analysis of the results, we encountered two important but distinct perspectives on medical education. On the one hand there is the perspective of the medical institution, which focusses on teaching complex clinical abilities to the clerks as well as on their role within group dynamics: "Professional identity formation is a term in medical education. This is about how doctors develop 
themselves, not only in knowledge and skills, but also as human being, because you want a doctor with good medical skills, who is also good in group dynamics and has social skills."

The second perspective was that of the educational institution, whose concerns with assessment and accountability gave the measurability of education an important role: "Education is such a huge machine and it needs to be accredited and the language spoken is checklists and prove it works. Effectiveness"

From the perspective of the medical institution there are many concerns about the different roles a clerk has to play in the event that they are also made to act as MedGezel. It was frequently mentioned that role confusion could arise among the clerks, which might hamper their education: "The clerks might be hesitant to coach the patient and to ask in depth questions in front of the doctor, because the clerk is the patient advocate in this setting, and the next moment he will be judged by this same doctor."

The other side of the coin of role confusion was also mentioned, where the physician might feel judged: "I think that the physicians had the idea that they would be judged, which is kind of peculiar, because normaly a clerk will sit next to them and will have some kind of judgement about them aswell."

Even though clerks are not allowed to give medical advice to patients in their normal role as clerk, one specialist mentioned: "I have the impression that the clerk might go and sit on the doctors chair and will answer all kinds of questions."

One of the experts from the quality department said that the reason for this fear might be rooted in the conviction of older physicians that what they do is fine, given that they have been doctors for many years and that this represents a kind of criticism on their work: "Some say: 'I'm a doctor for 30 years, I'm a fine doctor', though they do not listen that good and they are not willing to open up and learn to do better. It's a kind of autonomy in saying 'why? I'm doing well at my job."

Next to this perspective, it was mentioned that there are also different views from medical physicians on the importance of educational innovations. One of the participants mentioned that doctors value science within the medical field more than innovations within the field of management or education: "In practice you see that hard science and PhD trajectories are weighed much more heavily than soft projects within education".

From the perspective of the educational institution there seems to be a feeling that an innovation needs to be initiated by the institution itself. One specialist hypothesized that having the feeling of ownership needs to be deep-rooted in order to back up an innovation. This is particularly evident whenever an innovation comes from an external source and is not 'hospital-own'. "It's not really from OUR hospital. There are, I don't know how many FTEs of educators and doctors who develop education here, we can all do that ourselves and this all cost so much money that we are not going to do anything coming from elsewhere."

To summarize, there are concerns that the new role of the medical coach could break the traditional communion between specialists and clerks as well as with what this break could mean for the medical 
education.

\section{Alignment with the corporate strategies}

The analysis of the interviews revealed that an innovation can aid in realizing corporate strategies. A marketer at the quality department explained that it becomes increasingly important to distinguish a hospital from another providers of healthcare in order to improve its positioning on the market: "We use marketing to distinguish our hospital from other hospitals in the district." She explained that an innovation like MedGezel could help with this branding: "If you've defined your branding and your values, the innovation should fit that branding."

The same view was shared by the educational department, with one educator mentioning that an innovation can be used to distinguish a university from others: "At the selection of the universities one of the questions is: what characterizes your education? And where would you like to go?" She also said: "I think you can use MedGezel as an interpretation for the core values of this university." An expert on the quality department mentioned that MedGezel fits in the learning organization and the people orientated organization: "This fits seamlessly in the learning organization and the people orientated organization, in which you can explain 'people' in different contexts: as clerk, as patient, but also as medical specialist." $\mathrm{He}$ also mentioned that many policy plans have already been made years ago to reach organizational goals, including training patients and clerks in patient participation, and MedGezel might be a nice initiative to accomplish these goals: "This innovation touches many organizational goals in teaching patient participation and training the patient. These themes have been in policy plans for years, but that memo is gathering dust on the shelf."

\section{Discussion}

This study sheds light on the failed implementation of the MedGezel clinical education initiative, which intended to enhance the empathy skills of medical clerks by putting them into the role of medical coaches.

Current systems of care and the biomedical paradigm create a risk of a decline in empathy in healthcare (22). Both healthcare givers and patients may experience anxiety and stress because of the dehumanization of healthcare practices and processes (23). To overcome this, a choice was made to implement an empathy-based educational intervention. However, this intervention ultimately proved unsuccessful, begging the question of what went wrong.

We found a majority of participants in the study embraced the vision of the initiative, and while some argued the need for empathy training, others felt that existing empathic training and performance were already sufficient. It became evident that leadership was essential for the success of any such initiative and should be a focal part of planning activities. In one department, management and resources were no obstacle for implementation, while in others they were, despite the presence of a program coordinator. Participants expressed concerns about role confusion for clerks who not only had to play the role of a 
student, but also that of a patients' coach and advocate. Despite these challenges, the general alignment of the initiative with corporate strategy was reported to be fine.

Reporting of implementation successes or failures is uncommon in medical education and other practical examples of similar educational change initiatives are difficult to find in the literature. However, reflection on findings from literature dealing with change management and culture provided useful insights into the outcome of the MedGezel initiative.

On a personal level, we could recognize a struggle within the minds of the study participants. Most were keen on embracing the vision behind the initiative because it fit their moral compasses. At the same time, however, many were concerned that changing routines would take some effort and were tempted to maintain the status quo as a result. We observe this same struggle in the implementation of shared decision making (24). Moreover, literature suggest that health professionals overestimate the way they overcome their paternalistic attitude (25). The outcome of this struggle may have prevented participants from taking leadership and acting as change agents for future health care (13).

On an organizational level, we knew that the alignment with the current hospital vision was good. In many countries, shared decision making by doctors and patients is recognized as being a part of daily learning (26) and an empathic quest for patient preferences is considered appropriate for this process (27). In addition, the international Institute of Healthcare Improvement (IHI) encourages and supports more meaningful conversations between people who provide healthcare and people who receive it(28). Nevertheless, despite that organizational alignment, alignment with work floor routines was not perfect. Although all involved were there, i.e., the attending physician, the clerk and the patient, the clerk would in certain patient contacts focus on person-oriented histories instead of medical diagnoses. With focusing on these histories there will be a break in the traditional communion between physicians and clerks, which might ask for limited arrangements. Debriefing clerks and patients after consultations with physicians may have disrupted the work of the clerk. Such changes to practice routines may seem small, but study participants attributed considerable importance to them. Routine changes are known to be difficult and time consuming in medical education (16).

The most important Achilles heel of implementing the MedGezel initiative may have been a matter of professional culture. Putting the clerk in the role of medical coach and giving them the task of briefing and debriefing the patient around the physician consultation risked upgrading the status of the clerk and making the physician an object of potential criticism. A well-studied subject in medical culture is 'saving face' (29). Physician culture dictates that openly criticizing each other is something to be avoided and an open culture is needed to be able to discuss each other's performance (30). Studies of Witman (31) and Scheepers (32) in the Netherlands reveal flaws in the achievement of an open culture. Hierarchy issues also play a serious role and are part of our medical culture (33). This is quite evident in narratives of hierarchy in hospitals, where trainees were expected not to challenge or question the judgements or actions of their seniors (34). Habits, values, and attitudes, even dysfunctional ones, are seen as being part 
of one's identity and for a clerk to potentially challenge a senior would be considered as breaking unwritten rules (35).

\section{Strengths, limitations and future research}

The strength of this study is found in the effort to report on and learn lessons from a failure to successfully implement a medical training initiative. We interviewed a rich variety of stakeholders and analyzed the data through the perspective of readiness for change, which resulted in more clarity about the failure of the initiatives' implementation. A limitation is the small size of the study and the fact that the MedGezel program was executed in just one country. It would be most interesting to learn from implementation experiences from a variety of countries with different contexts and cultures. Moreover, we must address in more detail how to cope with the medical habitus when it comes to education and pedagogical improvements for future health. Several participants spoke about management issues that were in our judgement relatively easy to deal with. How could one recognize convenience arguments and distinguish them from issues that were more important? There is still a lot to learn about change management in our specific medical education context.

\section{Recommendations}

- Learn from mistakes and failures and publish the resulting insights;

- Stick to advice from theories of change. In our experience, several program implementations in our scope were not well planned (15);

- Spent energy on professional cultural issues when open culture is a pillar to build on or when hierarchy is at stake;

- Align with corporate strategy and prepare for hurdles when the workforce has different routines than the desired ones;

- Persist. A failure is not a reason to quit, but a reason to learn from mistakes and start all over with a better plan.

\section{Conclusion}

We introduced a novel form of clinical empathy education and believed that general positive reactions promised that it would be successfully implemented. Despite the work of an energetic coordinator, the program hit the 'wall of goodwill' and ultimately experienced an implementation failure. Investigating the causes of this revealed an internal struggle of participants who embraced the vision but did not follow through and act accordingly in practice. At the level of the organization, they lacked strong leadership and spoke about management hurdles that did not seem to be fatal for the program's failure. Rather, the most convincing explanation came from a professional cultural perspective in the form of an established medical culture with unwritten rules and rituals clashing with a new training initiative in which clerks were given a new role with more status through which they could critically observe the performance of their superiors. Despite this failure, we shall persist in our implementation efforts, only now with greater 
attention paid to the supervisors. Medical culture must be addressed in any future endeavor, which may require, for instance, framing participating physicians as courageous change agents for the benefit of future health care.

\section{Declarations}

\section{Ethical consideration}

All protocols were approved by the central Medical Research Ethics Committee United in Nieuwegein, the Netherlands (registration number W20.019) and the local Ethical Committee of the OLVG hospital of Amsterdam, the Netherlands (registration number W019.175). The participants participated voluntarily and were allowed to withdraw at any moment during the research without providing any reasons to ensure their privacy. All methods were carried out in accordance with relevant guidelines and regulations. Written informed consent was obtained from all the subjects and/or their legal guardian(s) before the interviews.

\section{Consent for publication}

Written informed consent was obtained by all the subjects and/or their legal guardian(s) before the interviews.

\section{Data availability statement}

The datasets generated and/or analyzed during the current study are not publicly available due to the personal nature of the interviews with the participants and the agreement that the interviews would not be retraceable to the person but are available from the corresponding author on reasonable request.

\section{Competing interests}

The authors declare that they have no competing interests.

\section{Funding}

No funding was received for this research.

\section{Authors contributions}

LB interviewed the participants. LB and GK wrote the first draft of the manuscript. All authors analyzed and interpreted the data obtained from the interviews. All authors had a major contribution in writing the main manuscript. All authors read and approved the final manuscript.

\section{Acknowledgements}

We acknowledge Mike Leach, being a native English speaker, for the linguistic check. 


\section{Abbreviations}

Not applicable

\section{Authors information}

The main researcher, BSc and PhD-candidate Luca Barak; the co-first-author and co-investigator, physician, curriculum designer and trainer of medical coaches Giliam Kuijpers; the second researcher, physician and manager of education, Lotte Hoeijmakers; and Fedde Scheele, a physician and an expert in change theory.

\section{References}

1. Hojat M, Vergare MJ, Maxwell K, Brainard G, Herrine SK, Isenberg GA, et al. The devil is in the third year: a longitudinal study of erosion of empathy in medical school. Acad Med. 2009 Sep;84(9):1182-91.

2. Branch WTJ, Kern D, Haidet P, Weissmann P, Gracey CF, Mitchell G, et al. The patient-physician relationship. Teaching the human dimensions of care in clinical settings. JAMA. 2001 Sep;286(9):1067-74.

3. Derksen F, Bensing J, Lagro-Janssen A. Effectiveness of empathy in general practice: a systematic review. Br J Gen Pract J R Coll Gen Pract. 2013 Jan;63(606):e76-84.

4. Kim SS, Kaplowitz S, Johnston M V. The effects of physician empathy on patient satisfaction and compliance. Eval Health Prof. 2004 Sep;27(3):237-51.

5. Fragkos KC, Crampton PES. The Effectiveness of Teaching Clinical Empathy to Medical Students: A Systematic Review and Meta-Analysis of Randomized Controlled Trials. Acad Med. 2020 Jun;95(6):947-57.

6. Witman Y. De medicus maatgevend Over leiderschap en habitus Regarding leadership and habitus. 2008;1-260.

7. Meiboom A, Diedrich C, Vries H De, Hertogh C, Scheele F. The hidden curriculum of the medical care for elderly patients in medical education: a qualitative study. Gerontol Geriatr Educ. 2015;36(1):3044.

8. Bourdieu P. opstellen over smaak, habitus en het veldbegrip. Amsterdam: Van Gennep. 1989.

9. Billett S. Learning through health care work: premises, contributions and practices. Med Educ. 2016 Jan;50(1):124-31.

10. Boor K, Scheele F, van der Vleuten CPM, Teunissen PW, den Breejen EME, Scherpbier AJJA. How undergraduate clinical learning climates differ: a multi-method case study. Med Educ. 2008 Oct;42(10):1029-36.

11. Pohontsch NJ, Stark A, Ehrhardt M, Kötter T, Scherer M. Influences on students' empathy in medical education: an exploratory interview study with medical students in their third and last year. BMC Med 
Educ. 2018 Oct;18(1):231.

12. Vijn TW, Fluit CRMG, Kremer JAM, Beune T, Faber MJ, Wollersheim H. Involving Medical Students in Providing Patient Education for Real Patients: A Scoping Review. J Gen Intern Med. 2017 Sep;32(9):1031-43.

13. Frenk J, Chen L, Bhutta ZA, Cohen J, Crisp N, Evans T, et al. Health professionals for a new century: transforming education to strengthen health systems in an interdependent world. Lancet (London, England). 2010 Dec;376(9756):1923-58.

14. Bank L, Jippes M, Leppink J, Scherpbier AJ, den Rooyen C, van Luijk SJ, et al. Are they ready? Organizational readiness for change among clinical teaching teams. Adv Med Educ Pract. 2017;8:807-15.

15. Bank L, Jippes M, van Rossum TR, den Rooyen C, Scherpbier AJJA, Scheele F. How clinical teaching teams deal with educational change: "we just do it”. BMC Med Educ. 2019 Oct;19(1):377.

16. Dagnone JD, Chan M-K, Meschino D, Bandiera G, den Rooyen C, Matlow A, et al. Living in a World of Change: Bridging the Gap From Competency-Based Medical Education Theory to Practice in Canada. Acad Med. 2020 Nov;95(11):1643-6.

17. Murphy K. From a Fumbled Beginning: If You Don't Make a Mistake, You Don't Make Anything. Trans Anal J [Internet]. 2012 Jan 1;42(1):28-33. Available from:

https://doi.org/10.1177/036215371204200104

18. Gray DE. Doing research in the real world. 2014. 729 p.

19. Westerman M, Teunissen PW, van der Vleuten CPM, Scherpbier AJJA, Siegert CEH, van der Lee N, et al. Understanding the transition from resident to attending physician: a transdisciplinary, qualitative study. Acad Med. 2010 Dec;85(12):1914-9.

20. Kennedy TJT, Lingard LA. Making sense of grounded theory in medical education. Med Educ. 2006 Feb;40(2):101-8.

21. Bank L, Jippes M, Scherpbier AJ, den Rooyen C, Scheele F. How To Get Your Clinical Teaching Team Ready For Curriculum Change: A Practical Guide. Adv Med Educ Pract. 2019;10:979-86.

22. Neumann M, Edelhäuser F, Tauschel D, Fischer MR, Wirtz M, Woopen C, et al. Empathy decline and its reasons: a systematic review of studies with medical students and residents. Acad Med. 2011 Aug;86(8):996-1009.

23. Zulueta $P$ de. Compassion in 21 st century medicine: Is it sustainable? Clin Ethics [Internet]. 2013 Sep 16;8(4):119-28. Available from: https://doi.org/10.1177/1477750913502623

24. Driever EM, Stiggelbout AM, Brand PLP. Shared decision making: Physicians' preferred role, usual role and their perception of its key components. Patient Educ Couns. 2020;103(1):77-82.

25. Ankolekar A, Steffensen KD, Olling K, Dekker A, Wee L, Roumen C, et al. Practitioners' views on shared decision-making implementation: A qualitative study. PLoS One [Internet]. 2021;16(November):1-18. Available from: http://dx.doi.org/10.1371/journal.pone.0259844 
26. Spinnewijn L, Aarts J, Verschuur S, Braat D, Gerrits T, Scheele F. Knowing what the patient wants: a hospital ethnography studying physician culture in shared decision making in the Netherlands. BMJ Open. 2020 Mar;10(3):e032921.

27. Sackett DL, Rosenberg WM, Gray JA, Haynes RB, Richardson WS. Evidence based medicine: what it is and what it isn't. Vol. 312, BMJ (Clinical research ed.). 1996. p. 71-2.

28. Institute for Healthcare Improvement. Person- and family-centered care [Internet]. 2021. Available from: http://www.ihi.org/Topics/PFCC/Pages/default.aspx

29. Ginsburg S, van der Vleuten C, Eva KW, Lingard L. Hedging to save face: a linguistic analysis of written comments on in-training evaluation reports. Adv Health Sci Educ Theory Pract. 2016 Mar;21(1):175-88.

30. Malik RF, Buljac-Samardžić M, Amajjar I, Hilders CGJM, Scheele F. Open organisational culture: what does it entail? Healthcare stakeholders reaching consensus by means of a Delphi technique. BMJ Open. 2021 Sep;11(9):e045515.

31. Witman Y. What do we transfer in case discussions? The hidden curriculum in medicine... Perspect Med Educ. 2014 Apr;3(2):113-23.

32. Scheepers RA, van den Goor M, Arah OA, Heineman MJ, Lombarts KMJMH. Physicians' Perceptions of Psychological Safety and Peer Performance Feedback. J Contin Educ Health Prof. 2018;38(4):250-4.

33. Brennan PA, Davidson M. Improving patient safety: we need to reduce hierarchy and empower junior doctors to speak up. BMJ. 2019 Jul;366:I4461.

34. Crowe S, Clarke N, Brugha R. "You do not cross them": Hierarchy and emotion in doctors' narratives of power relations in specialist training. Soc Sci Med. 2017 Aug;186:70-7.

35. Heifetz RA, Linsky M. A survival guide for leaders. Harv Bus Rev. 2002 Jun;80(6):65-74,152.

36. Alders I, Henselmans I, Smits C, Visscher T, Heijmans M, Rademakers J, et al. Patient coaching in specialist consultations. Which patients are interested in a coach and what communication barriers do they perceive? Patient Educ Couns. 2019 Aug;102(8):1520-7.

37. Goodyear-Smith F, Buetow S. Power issues in the doctor-patient relationship. Health Care Anal. 2001;9(4):449-62. 